\title{
Macrophages promote the growth and invasion of endometrial stromal cells by downregulating IL-24 in endometriosis
}

\author{
Jun Shao ${ }^{1, *}$, Bing Zhang ${ }^{1, *}$, Jia-Jun Yu ${ }^{1}$, Chun-Yan Wei ${ }^{1}$, Wen-Jie Zhou ${ }^{1,2}$, Kai-Kai Chang ${ }^{1,3}$, \\ Hui-Li Yang ${ }^{1}$, Li-Ping Jin ${ }^{4}$, Xiao-Yong Zhư ${ }^{1,3}$ and Ming-Qing Li ${ }^{1,2,3}$ \\ ${ }^{1}$ Laboratory for Reproductive Immunology, Hospital of Obstetrics and Gynecology, ${ }^{2}$ Key Laboratory of \\ Reproduction Regulation of NPFPC, SIPPR, IRD, Fudan University, Shanghai, People's Republic of China, \\ ${ }^{3}$ Shanghai Key Laboratory of Female Reproductive Endocrine Related Diseases, Shanghai, People's Republic of \\ China and ${ }^{4}$ Clinical and Translational Research Center, Shanghai First Maternity and Infant Hospital, \\ Tongji University School of Medicine, Shanghai, People's Republic of China
}

Correspondence should be addressed to M-Q Li or X-Y Zhu; Email: mqli@fudan.edu.cn or zhuxiaoyong@fudan.edu.cn

*(J Shao and B Zhang contributed equally to this work)

\begin{abstract}
Macrophages play an important role in the origin and development of endometriosis. Estrogen promoted the growth of decidual stromal cells (DSCs) by downregulating the level of interleukin (IL)-24. The aim of this study was to clarify the role and mechanism of IL-24 and its receptors in the regulation of biological functions of endometrial stromal cells (ESCs) during endometriosis. The level of IL-24 and its receptors in endometrium was measured by immunohistochemistry. In vitro analysis was used to measure the level of IL-24 and receptors and the biological behaviors of ESCs. Here, we found that the expression of IL-24 and its receptors (IL-20R1 and IL-20R2) in control endometrium was significantly higher than that in eutopic and ectopic endometrium of women with endometriosis. Recombinant human IL-24 (rhIL-24) significantly inhibited the viability of ESCs in a dosage-dependent manner. Conversely, blocking IL-24 with anti-IL-24 neutralizing antibody promoted ESCs viability. In addition, rhIL-24 could downregulate the invasiveness of ESCs in vitro. After co-culture, macrophages markedly reduced the expression of IL-24 and IL-20R1 in ESCs, but not IL-22R1. Moreover, macrophages significantly restricted the inhibitory effect of IL-24 on the viability, invasion, the proliferation relative gene $\mathrm{Ki}-67$, proliferating cell nuclear antigen (PCNA) and cyclooxygenase2 (COX-2), and the stimulatory effect on the tumor metastasis suppressor gene CD82 in ESCs. These results indicate that the abnormally low level of IL-24 in ESCs possibly induced by macrophages may lead to the enhancement of ESCs' proliferation and invasiveness and contribute to the development of endometriosis.

Reproduction (2016) 152 673-682
\end{abstract}

\section{Introduction}

Endometriosis is a common estrogen-dependent inflammatory condition, which is characterized by the growth of endometriotic lesions consisting of endometrial glands and stroma outside the uterus and is associated with pelvic pain and infertility (Giudice 2010). The exact prevalence of endometriosis is unknown but is estimated to range from 2 to $10 \%$ of reproductive age women, and rise up to $50 \%$ of infertile women (Meuleman et al. 2009). The precise etiology and pathogenesis of endometriosis still remain controversial despite extensive research. Among these, the most widely accepted theory is Sampson's theory of implantation of endometrial cells and fragments refluxed through the fallopian tubes during the menstrual period (Sampson 1927). According to this theory, a puzzle emerges that only $6-10 \%$ of women develop endometriosis, whereas retrograde menstruation is observed in most women, suggesting that other factors may also trigger the formation of endometriotic lesions, such as hormones, cytokines, growth factors, angiogenic factors and cancer-related molecules (Giudice \& Kao 2004).

Immunocytes are thought to play an important role in the origin and development of endometriosis. The dysfunction of immune cells and their mediators are responsible for the poor clearance of ectopic endometrium. Moreover, they promote ectopic implantation and growth of endometrium. Macrophages, which are recruited to the peritoneal cavity and mainly present M2 phenotype, account for $80 \%$ of all lymphocytes in the peritoneal fluid. Some studies have shown that the total number, concentration and activated status of peritoneal macrophages are higher in 
women with endometriosis (Haney et al. 1981, Halme et al. 1984, Halme et al. 1987, Dunselman et al. 1988).

Melanoma differentiation-associated gene-7 (mda-7)/ interleukin-24 (IL-24), a member of IL-10 gene family, is a newly discovered tumor-suppressing protein (Sauane et al. 2003). It can bind to its two different heterodimeric receptor complexes, IL-20R1/IL-20R2 and IL-22R/IL-20R2 (Dumoutier et al. 2001). As a dualacting cytokine, IL-24 participates in normal immune response as a cytokine at normal physiological levels. At supraphysiological levels, IL-24 displays prominent anti-tumor biological properties such as promoting the apoptosis of tumor cells, inhibiting the angiogenesis and the invasion of tumor cells and enhancing the sensitivity to radiotherapy, without affecting normal cells (Fisher et al. 2003, Su et al. 2003, Sauane et al. 2008). MDA-7/ IL-24 has now proven efficacious and secure in a phase I/II clinical trial in humans with multiple advanced cancers (Dent et al. 2010). Our previous study showed that DSCs expressed IL-24 and its receptors in human during early pregnancy, and estrogen promoted the growth of DSCs by downregulating the level of IL-24 (Shao et al. 2013).

Although endometriosis is a benign estrogendependent disease, endometriosis and cancer are similar in several aspects such as uncontrolled cell growth and invasion (Ueda et al. 2002). Therefore, this study was undertaken to clarify the role and mechanism of IL-24 and its receptors (IL-20R1, IL-20R2 and IL-22R1) in biological functions' regulation of ESCs induced by cross-talking between macrophages and ESCs.

\section{Materials and methods}

\section{Tissue collection}

This study was approved by the Ethical Committee of the Obstetrics and Gynecology Hospital, Fudan University. All tissue samples were obtained with the consent of the patients. The study's subjects were women of reproductive age attending the Obstetrics and Gynecology Hospital of Fudan University between March 2015 and January 2016. By laparoscopy and histological analysis, endometriosis was confirmed. According to the revised American Fertility Society classification of endometriosis, all the women with endometriosis were classified as stages III/IV (American Fertility Society 1985), and none of the women had received hormonal medication in the 6 months before the surgical procedure. Endometriotic cyst wall tissues (ectopic endometrium) $(n=10)$ were obtained from women between ages 23 and 46 years during surgeries. The endometrial tissues were obtained from fertile women (ages $25-45$ years) with $(n=38)$ or without $(n=10)$ endometriosis as control. All the samples were confirmed histologically according to the established criteria. The tissues used for immunohistochemistry were obtained during both the proliferative and secretory phases of the cycle, but for all other experiments, the samples were collected only in the proliferative phase of the cycle.
The endometrial tissues from women with endometriosis were collected under sterile conditions and transported to the laboratory on ice in Dulbecco's modified Eagle's medium (DMEM)/F-12 (Gibco) with 10\% fetal calf serum (FCS; Hyclone, Logan, UT, USA).

Peripheral blood samples $(15 \mathrm{~mL})$ from the healthy women were taken sterilely in heparinized Hank's buffer solution (Gibco) before the administration of anesthesia. The samples were immediately transported to a laboratory on ice for bead sorting of the monocytes.

\section{Immunohistochemistry}

Paraffin sections $(5 \mu \mathrm{m})$ of the endometriotic cyst wall tissue and eutopic endometrial tissues from women with or without endometriosis in the proliferative or secretory phase of the cycle were dehydrated in graded ethanol and incubated with $3 \%$ hydrogen peroxide in $1 \%$ bovine serum albumin in Trisbuffered saline (TBS) to block endogenous peroxidase. The samples were then incubated with mouse anti-human IL-24 monoclonal antibody $(25 \mu \mathrm{g} / \mathrm{mL}$; R\&D Systems), IL-20R1 antibody $(15 \mu \mathrm{g} / \mathrm{mL} ;$ R\&D Systems), IL-20R2 antibody (SC99085; 1:100; Santa Cruz Biotechnology), IL-22R1 antibody $(15 \mu \mathrm{g} / \mathrm{mL}$; R\&D Systems) or mouse/rabbit IgG isotype (SinoAmerica Co, Ltd, Shanghai, China) overnight at $48^{\circ} \mathrm{C}$ in a humid chamber. After washing three times with TBS, the sections were overlaid with peroxidase-conjugated goat anti-mouse/ rabbit IgG (Golden Bridge International, Inc, Beijing, China), and the reaction was developed with 3,3-diaminobenzidine and counterstained with hematoxylin. The experiments were repeated three times with 12 different samples.

\section{Cell isolation and culture}

According to the previous methods (Li et al. 2012, Mei et al. 2014), the ESCs of eutopic endometrium from women with endometriosis (eutopic ESCs) and peripheral monocytes were isolated. For isolation of ESCs, the endometrial tissues were digested with collagenase type IV (0.1\%; Sigma) for $30 \mathrm{~min}$ at $37^{\circ} \mathrm{C}$ with constant agitation for recovering ESCs. To remove cellular debris, the tissue pieces were filtered through sterile gauze pads (pore diameter sizes: 200 mesh). After gentle centrifugation, the supernatant was discarded, and the cells were re-suspended in DMEM/F-12. The ESCs were separated from epithelial cells by passing them over sterile gauze pads (pore diameter sizes: 400 mesh). The filtrated suspension was layered over Ficoll and centrifuged at $800 \mathrm{~g}$ for $20 \mathrm{~min}$ to further remove leukocytes and erythrocytes, and the middle layer was collected and then washed with D-Hanks solution. The ESCs were placed in a culture flask and allowed to adhere for $20 \mathrm{~min}$. The adherent stromal cells were cultured as monolayer in flasks with DMEM/F-12 supplemented with $10 \% \mathrm{FCS}$ and $20 \mathrm{mmol} / \mathrm{L} \mathrm{HEPES}$ and incubated in a humidified incubator with $5 \% \mathrm{CO}_{2}$ at $37^{\circ} \mathrm{C}$. These methods supplied $>98 \%$ Vimentin-positive (Vimentin ${ }^{+}$) ESCs.

For isolation of monocytes, peripheral blood mononuclear cells (PBMC) were isolated from the healthy fertile women's blood samples by Ficoll-Hypaque density gradient centrifugation. According to the manufacturer's instructions, 
$\mathrm{CD}_{14}^{+}$monocytes were purified from PBMC by magnetic affinity cell sorting (MACS) using the human CD14 MicroBeads kit (Miltenyi Biotec $\mathrm{GmbH}$, Germany). The purity of $\mathrm{CD}_{14} 4^{+}$monocytes was $>95 \%$ as determined by flow cytometry. Subsequently, these monocytes were differentiated to macrophages in vitro according to previous method (Mei et al. 2014). Specifically, the monocytes were stimulated with granulocyte macrophage colony-stimulating factor (GM-CSF, $5 \mathrm{ng} / \mathrm{mL}$; R\&D Systems) and macrophage colony-stimulating factor (M-CSF, $20 \mathrm{ng} / \mathrm{mL}$; R\&D Systems) in RPMl-1640 medium (Gibco) containing 10\% FBS and $2 \mathrm{mM}$ L-glutamine for up to 6 days. The medium that contained GM-CSF and M-CSF was changed every 3 days. After 6 days, the monocyte-derived macrophages were further incubated with ESCs in contact co-culture units for another 2 days.

\section{Bromodeoxyuridine (BrdU) cell proliferation and Matrigel invasion assays}

The isolated eutopic ESCs from women with endometriosis were treated with recombinant human IL-24 (rhIL-24, at 0, 1, 10 or $100 \mathrm{ng} / \mathrm{mL}$ ) or anti-IL-24 neutralizing antibody ( $\alpha$-IL-24, at $0,0.05,0.5$, or $5 \mu \mathrm{g} / \mathrm{mL}$ ) for 24 or $48 \mathrm{~h}$. In addition, mouse isotype $(1 \mathrm{mg} / \mathrm{mL})$ (Sino-America Co, Ltd) or media as a negative

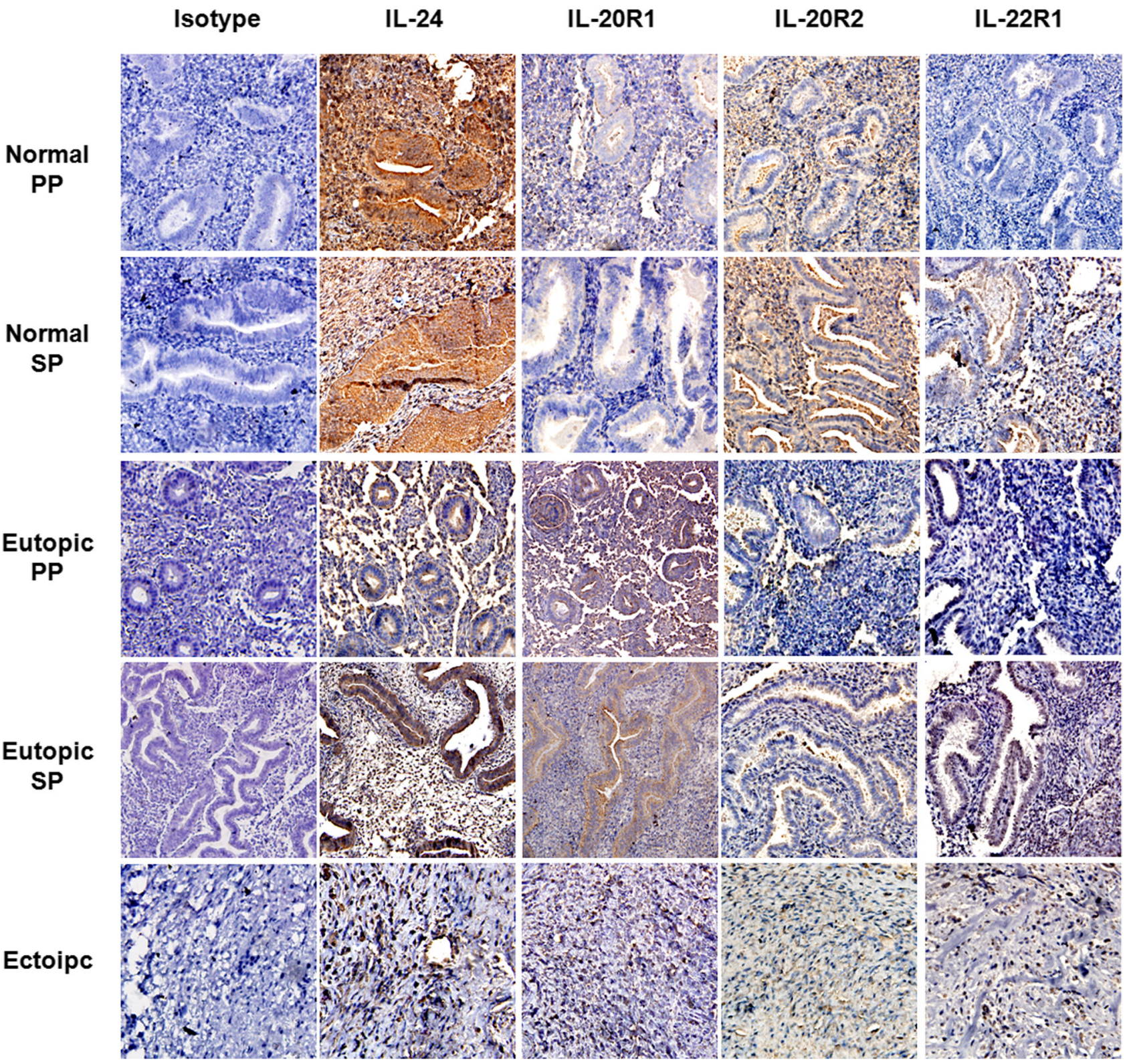

Figure 1 The expression of IL-24 is decreased in the eutopic and ectopic endometrium of women with endometriosis. The expression of IL-24 and its receptors (IL-20R1, IL-20R2 and IL-22R1) in the endometrium of women without endometriosis $(n=10)$, eutopic endometrium $(n=10)$ and ectopic lesion $(n=10)$ from women with endometriosis were analyzed by immunohistochemistry. Original magnification: $\times 200$. Normal: endometrium of women without endometriosis; eutopic: endometrium of patient with endometriosis; ectopic: endometriotic tissues; PP, proliferative phase; SP, secretory phase. 

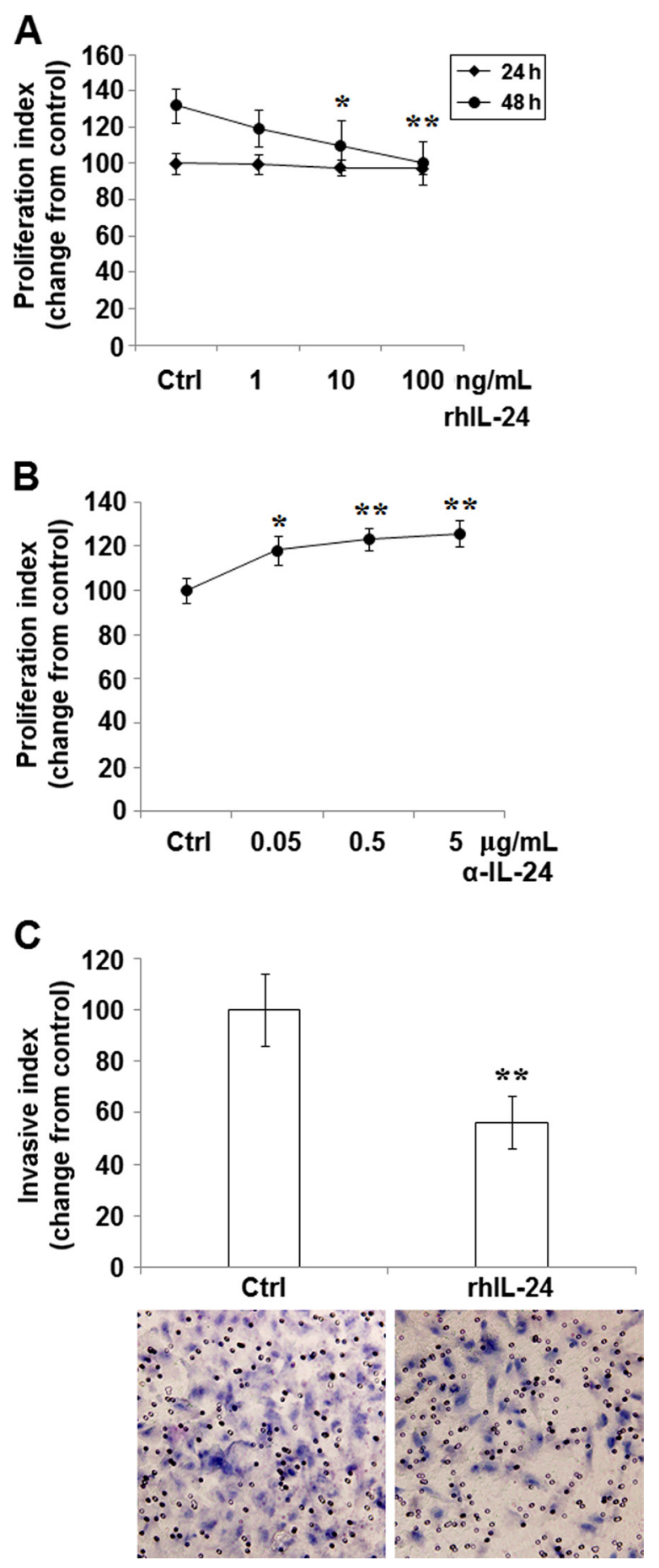

Figure 2 IL-24 suppresses the proliferation and invasiveness of ESCs. Primary ESCs $(n=6)$ from eutopic endometrium with endometriosis were incubated with different concentrations of rhIL-24 (0, 1, 10 and $100 \mathrm{ng} / \mathrm{mL}$ for (A); $100 \mathrm{ng} / \mathrm{ml}$ for (c)), anti-IL-24 neutralizing antibody $(0,0.05,0.5$ and $5 \mu \mathrm{g} / \mathrm{mL})$ for $48 \mathrm{~h}$, the mouse isotype or media were added to some wells as a negative control, and then BrdU proliferation assay (A and B) and Matrigel invasion assay (C) were used to detect the proliferation and invasiveness of ESCs. The invasion index was calculated as (the ratio of the cells number migrated to the lower surfaces in each group/the vehicle control)*100. Original magnification: $\times 200$. Data are expressed as the mean \pm S.E.M. $* P<0.05$ or ${ }^{* *} P<0.01$ compared with the control. control was added. Then the ability of ESCs proliferation was detected by BrdU cell proliferation assay kits (Millipore) according to the manufacturer's instruction. The experiments were performed in triplicate, and repeated four times.

In addition, the invasion of the eutopic ESCs from women with endometriosis was analyzed by Matrigel invasion assay, following our previous procedure (Li et al. 2012). Briefly, the cell inserts $(8 \mu \mathrm{m}$ pore size, $6.5 \mathrm{~mm}$ diameter, Corning) coated with 15-25 $\mu \mathrm{L}$ Matrigel were placed in a 24-well plate. The ESCs of $2 \times 10^{4}$ were plated in the upper chamber. Recombinant human IL-24 protein $(100 \mathrm{ng} / \mathrm{mL})$ or the vehicle was added. The lower chamber was filled with $800 \mu \mathrm{L}$ medium.
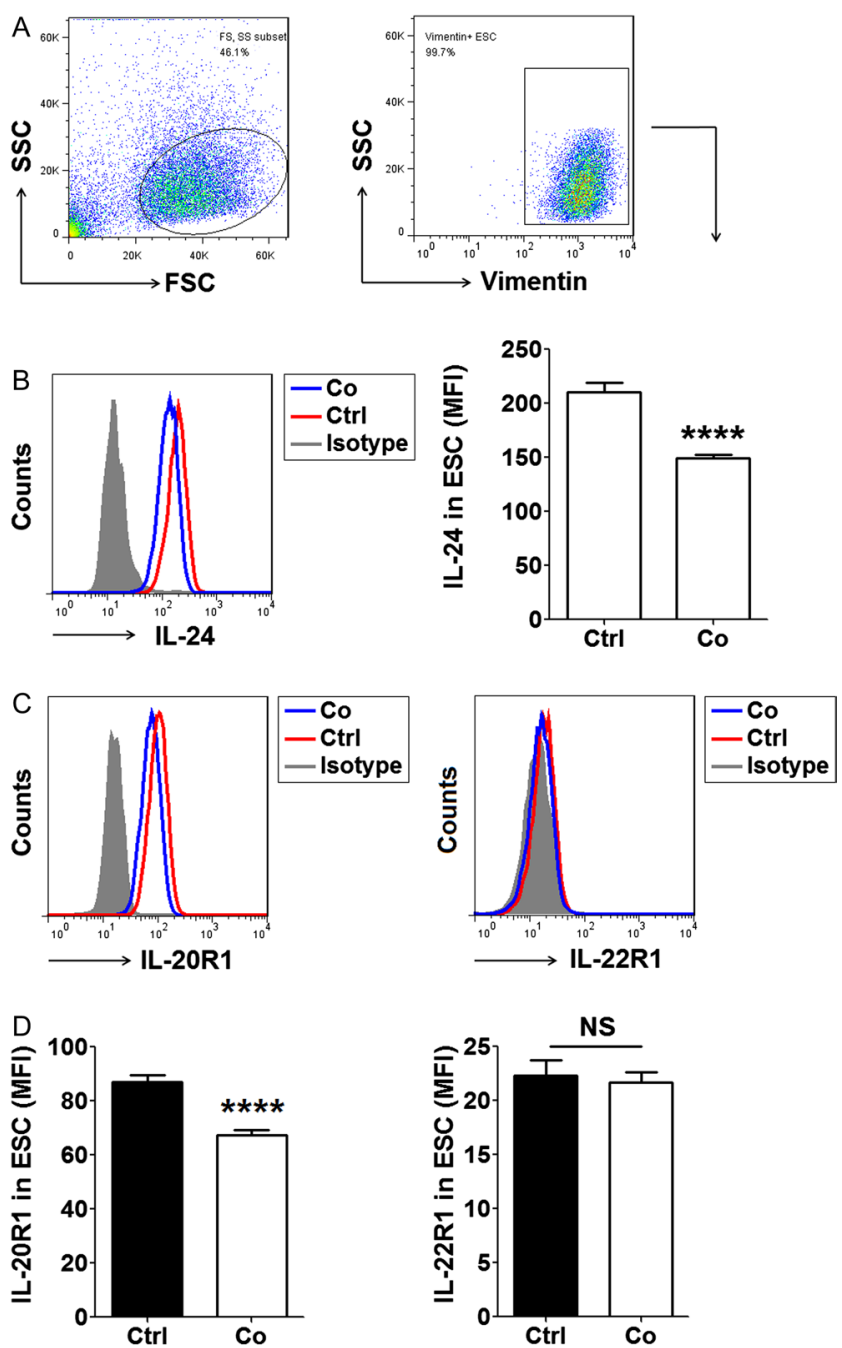

Figure 3 Macrophages downregulate the expression of IL-24 and IL-20R1 by ESCs. After direct co-culture between eutopic ESCs $(n=6)$ and human monocytes-derived macrophages from the peripheral blood of healthy women $\left(1 \times 10^{5}\right.$ cells/well, respectively) $(\mathrm{A})$ for $48 \mathrm{~h}$, the FCM was used to analyze the expression of IL-24 (B) and its receptors IL-20R1 (C and D left) and IL-22R1 (C and D right) in Vimentin ${ }^{+}$ESCs (A). ESCs cultured alone in the same media were used as the control. MFI: mean fluorescence intensity. Co: co-culture of ESCs and monocytes-derived macrophages. Isotype: isotypic control antibodies. Data are expressed as the mean \pm S.E.M. ${ }^{* * * *} P<0.0001$ compared with the control. NS, no significant difference. 


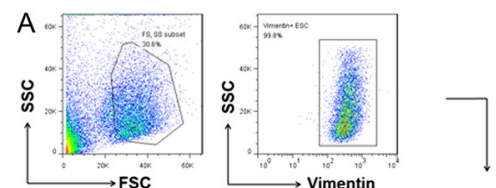

B
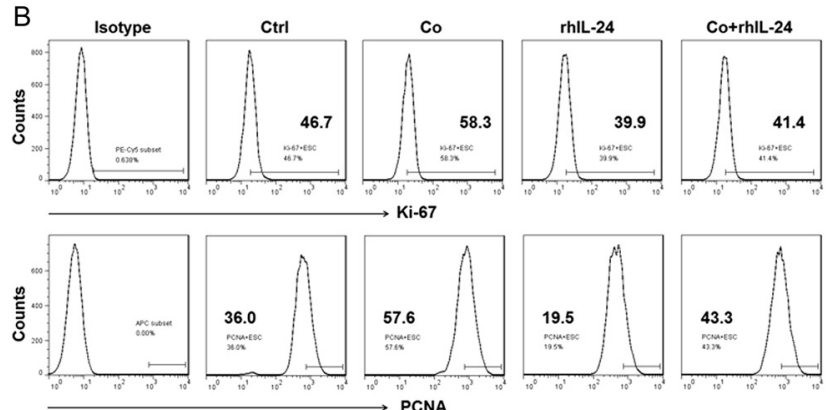

The cells were then incubated at $37^{\circ} \mathrm{C}$ for $48 \mathrm{~h}$. The inserts were removed, washed in PBS and the non-invading cells together with the Matrigel were removed from the upper surface of the filter by wiping with a cotton bud. The inserts were then fixed in methanol for $10 \mathrm{~min}$ at room temperature and stained with hematoxylin. The result was observed under Olympus BX51+P70 microscope (Olympus). The cells that migrated to the lower surfaces were counted in five predetermined fields

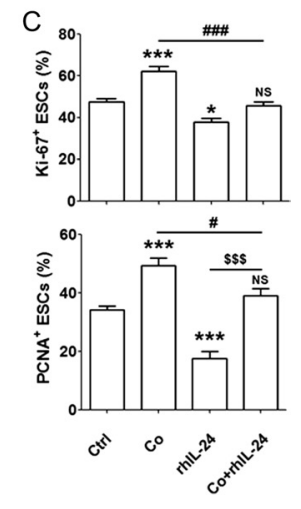

Figure 4 The effect of macrophages on $\mathrm{Ki}-67$ and PCNA expression of ESCs is dependent on IL-24. The eutopic ESCs $(n=6)$ from women with endometriosis were co-cultured with or without monocytes plus rhIL-24 $(100 \mathrm{ng} / \mathrm{mL})$ or not for $48 \mathrm{~h}$, respectively. Then the expression of Ki-67 and PCNA in Vimentin ${ }^{+}$ESCs (A) was detected by FCM (B and $\mathrm{C})$, respectively. Co: co-culture of ESC and monocytes-derived macrophages. Co+rhlL-24: co-culture of ESC and monocytes-derived macrophages plus rhIL-24. All data are expressed as the mean \pm S.E.M. ${ }^{*} P<0.05$ or $* * * P<0.001$ compared with control; ${ }^{\sharp} P<0.05$ or $\# \# P<0.001$ compared with co-culture group; $\$ \$ P<0.001$ compared with rhIL-24 alone. NS, no significant difference.

at a magnification of $\times 200$. Each experiment was carried out in triplicate, and repeated three times.

\section{Flow cytometry (FCM)}

The eutopic ESCs from women with endometriosis were cultured in 24-well plates (Corning) at a density of $1 \times 10^{5}$ cells/ well. In the contact co-culture unit, the monocytes-derived
A
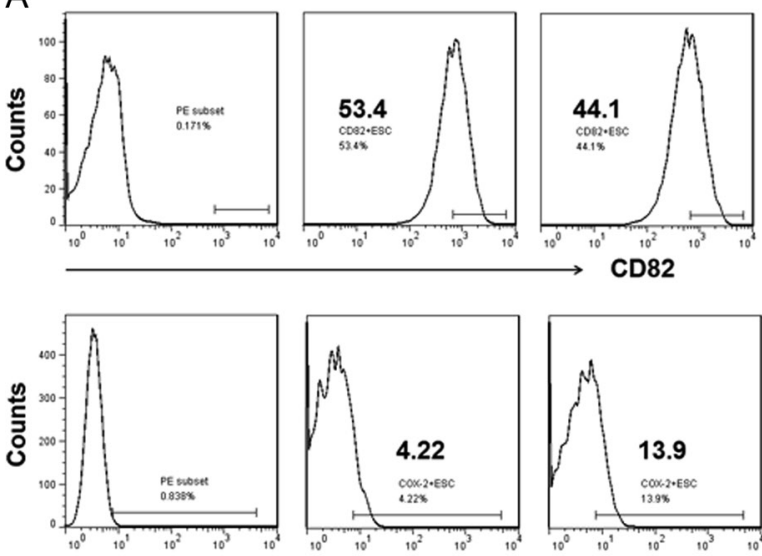

cox-2
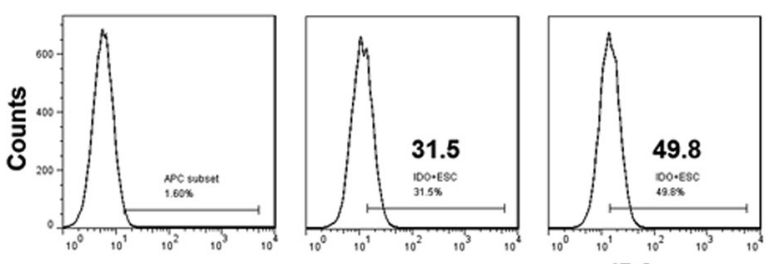

IDO
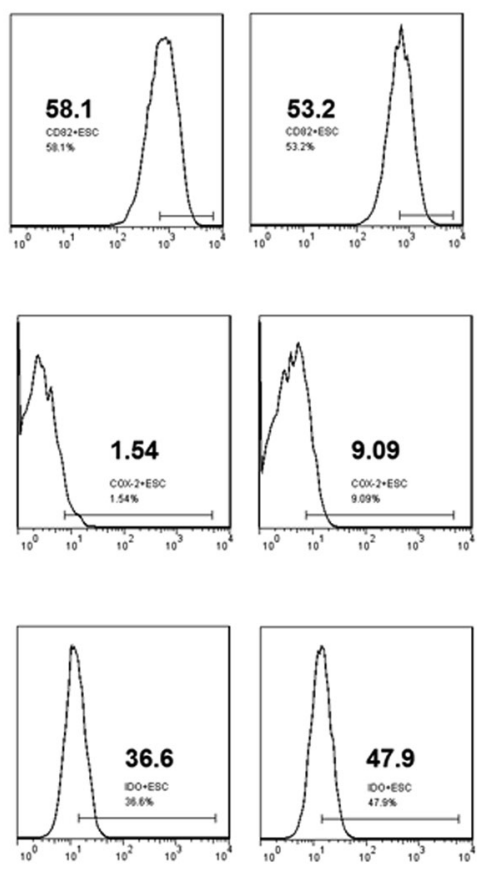
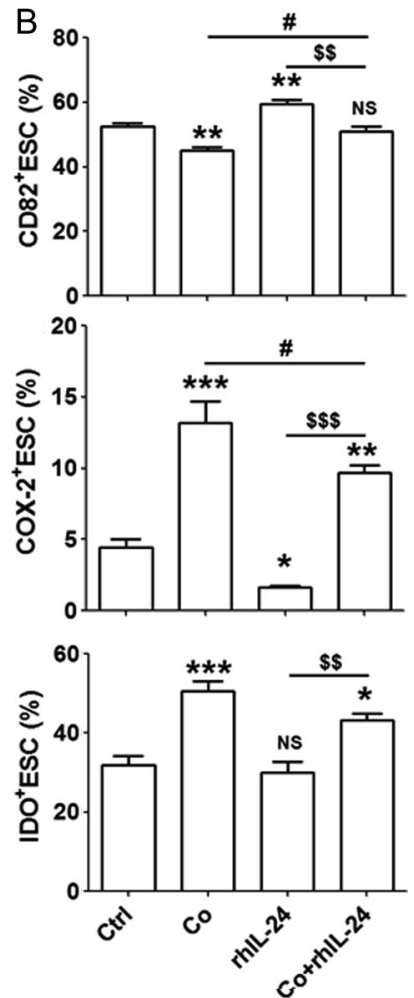

Figure 5 The effect of macrophages on COX-2 and CD82 expression of ESCs is dependent on IL-24. The ESCs $(n=6)$ were treated as described in Fig. 4, then the expression of CD82, COX-2 and IDO in Vimentin ${ }^{+}$ESCs was detected by FCM (A and B), respectively. All data are expressed as the mean \pm S.E.M. ${ }^{*} P<0.05,{ }^{* *} P<0.01$ or ${ }^{* * *} P<0.001$ compared with control; ${ }^{\sharp} P<0.05$ compared with co-culture group; ${ }^{\$ \$} P<0.01$ or ${ }^{\$ \$} P<<0.001$ compared with rhIL-24 alone. 
macrophages from peripheral blood of healthy women were directly added to the wells at the same density as the ESCs, and further stimulated with or without rhIL-24 $(100 \mathrm{ng} / \mathrm{mL})$ for another $48 \mathrm{~h}$. The cultured eutopic ESCs alone in the same media were used as the control. After co-culture, the expression of IL-24 (R\&D Systems), IL-20R1 (R\&D Systems), IL-22R1 (R\&D Systems), Ki-67 (BioLegend, USA), PCNA (BioLegend, San Diego, CA, USA), CD82 (BioLegend), COX-2 (Cell Signaling Technology) and Indoleamine 2,3-dioxygenase-1 (IDO1) (Cell Signaling Technology) on Vimentin ${ }^{+}$ESCs (R\&D Systems) was analyzed by flow cytometry, and the corresponding isotypic control antibodies were used.

\section{Statistics}

All values are shown as mean \pm S.E.M. The data were analyzed by a $t$-test in the case of two groups or by a one-way ANOVA using Tukey's post hoc test in multiple groups (SPSS, version 11.5). The differences were considered as statistically significant at $P<0.05$.

\section{Results}

The expression of IL-24 is decreased in the eutopic and ectopic ESCs from women with endometriosis

To investigate whether $\mathrm{IL}-24$ and its receptors are expressed in ESCs, we compared the expression level of IL-24 and its receptors (IL-20R1, IL-20R2 and IL-22R1) proteins in the endometrium with or without endometriosis. As depicted in Fig. 1, we found that the expression of IL-24 and its receptor IL-20R2 in normal endometrium was significantly higher than that in eutopic and ectopic endometrium from women with endometriosis. Moreover, the positive expression of IL-24 and IL-20R2 was observed in both endometrial stromal and epithelial cells. However, IL-22R1 expression in all endometrium tissues was very low. There was the highest level of IL-20R1 in eutopic endometrium from women with endometriosis. These results suggest that the lower expression of IL-24 and its receptor IL-20R2 in eutopic and ectopic endometrium stromal cells may participate in regulating the biological behaviors of ESCs, and further be involved in the origin and development of endometriosis.

\section{IL-24 suppresses the proliferation and invasiveness of ESCS}

To further probe the role of $\mathrm{IL}-24$ in regulating biological behavior of ESCs, we evaluated its effect on proliferation and invasiveness of ESCs in vitro. As shown in Fig. 2, rhIL-24 significantly decreased the proliferation of the ESCs after treatment for $48 \mathrm{~h}$ $(P<0.05$ or $P<0.01)$, especially at the concentration of $100 \mathrm{ng} / \mathrm{mL}$ (Fig. 2A). Conversely, blocking IL-24 with anti-human IL-24 antibodies ( $\alpha$-IL-24) notably promoted the proliferation of ESCs $(P<0.05$ or $P<0.01$ ) (Fig. 2B). Meanwhile, rhlL-24 obviously suppressed the invasiveness of ESCs $(P<0.01)$ (Fig. 2C). Our results suggest that IL-24 in vitro suppresses the proliferation and invasion of ESCs in an autocrine manner.
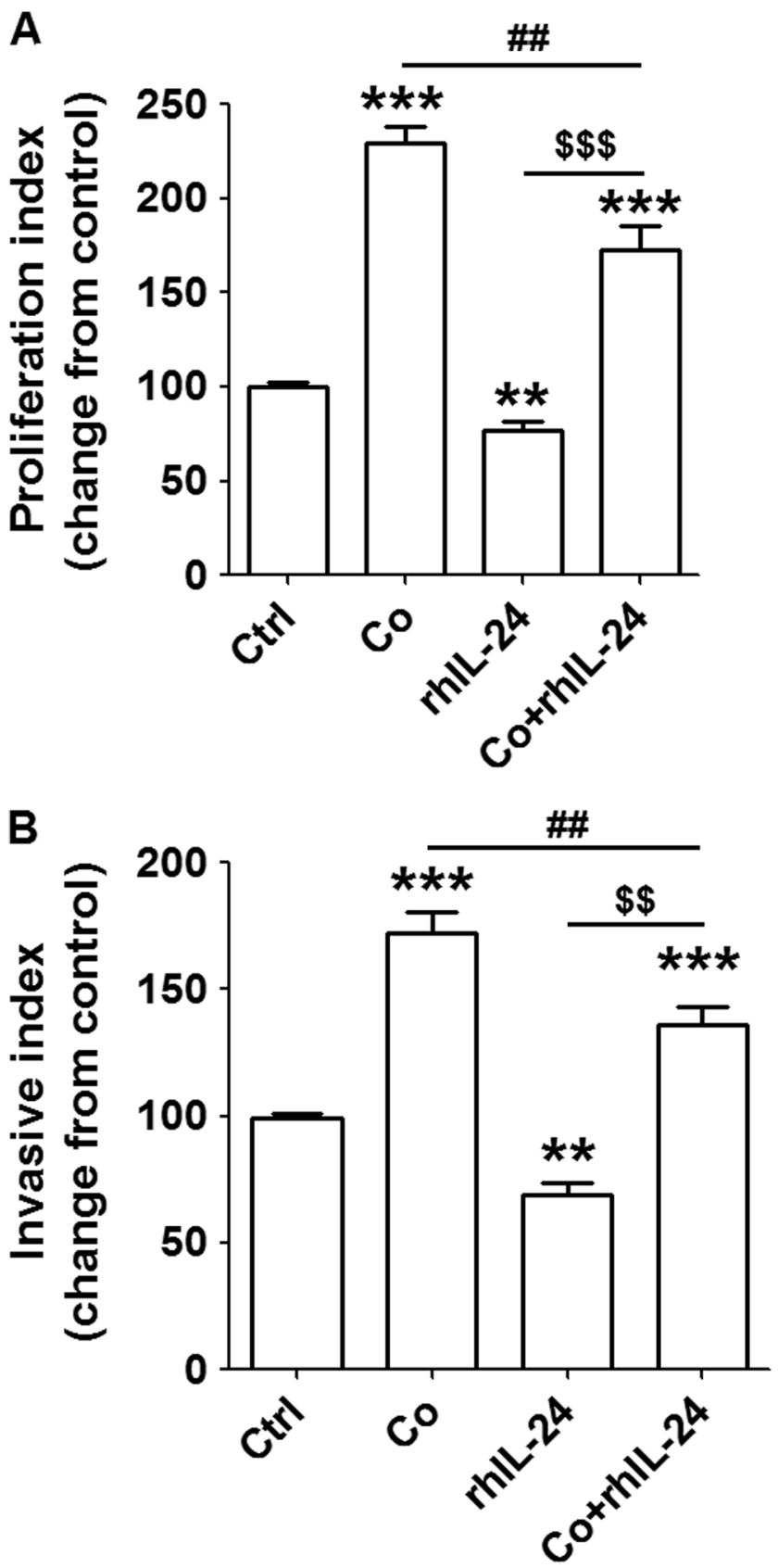

Figure 6 Macrophages promote the viability and invasion of ESCs partly by IL-24 suppression. The ESCs $(n=6)$ were treated as described in Fig. 4, then BrdU proliferation (A) and Matrigel invasion (B) were performed to examine the proliferation and invasiveness in ESCs. All data are expressed as the mean \pm S.E.M. ${ }^{* *} P<0.01$ or ${ }^{* * *} P<0.001$ compared with control; ${ }^{\# \#} P<0.01$ compared with co-culture group; $\$ P<0.01$ or $\$ \$ P<0.001$ compared with rhlL-24 alone. 


\section{Macrophages downregulate the expression of IL-24 and IL-20R1 in ESCS}

To evaluate whether macrophages regulate the expression of IL-24 and its receptors in ESCs, we constructed a contact co-culture model with Vimentin ${ }^{+}$ eutopic ESC from endometriosis and human monocytesderived macrophages (Fig. 3A). As shown, co-culture with macrophages led to low levels of IL-24 (Fig. 3B) and IL-20R1 (Fig. 3C and D left) in ESCs $(P<0.0001)$. However, there was no difference in IL-22R1 expression $(P>0.05)$ (Fig. 3C and D left). The data indicate that macrophages in endometriotic milieu may regulate biological behaviors of ESCs by downregulating IL-24 and its receptor.

\section{The effect of macrophages on Ki-67, PCNA, COX-2 and CD82 expression of ESCs is dependent on IL-24}

It is reported that COX-2 (Matsuzaki et al. 2004, Kao et al. 2011), IDO1 (Mei et al. 2013) and CD82 (Li et al. 2011) are involved in modulating the proliferation and or invasiveness of ESCs. To investigate the role of IL-24 and macrophages on these proliferation-related and invasion-related molecules in ESCs (Fig. 4A), ESCs were treated with or without rhIL-24 after Co-culture with or without macrophages. As shown in Figs 4 and 5, macrophages significantly upregulated the expression of Ki-67, PCNA and COX-2 and downregulated the expression of CD82 in ESCs in co-culture units $(P<0.01$ or $P<0.001)$ (Figs $4 \mathrm{~B}, \mathrm{C}$ and $5 \mathrm{~A}, \mathrm{~B})$. However, exposure with rhIL-24 resulted in an opposite effect on these molecules in ESCs $(P<0.05$, $P<0.01$ or $P<0.001$ ) (Figs $4 \mathrm{~B}, \mathrm{C}$ and $5 \mathrm{~A}, \mathrm{~B}$ ). In addition, rhIL-24 could partly or completely reverse the effect of macrophages on Ki-67, PCNA, CD82 and COX-2 expression (Figs 4B, C and 5A, B).

\section{Macrophages promotes the viability and invasion of ESCs partly by IL-24 suppression}

Finally, to investigate the impact of macrophages on ESCs behavior and to explore whether this effect is dependent on regulation of IL-24, BrdU proliferation and Matrigel invasion assays were performed to examine the proliferation and invasiveness of ESCs respectively. Consistent with the level of proliferation-related and invasion-related molecules, macrophages could markedly promote the proliferation and invasiveness of ESCs in ESCs-macrophages co-culture unit $(P<0.001)$ (Fig. 6A and B). Treatment with IL-24 inhibited the proliferation and invasiveness of ESCs $(P<0.01)$ (Fig. 6A and $\mathrm{B}$ ), and partly downregulated the stimulatory effect of macrophages on the viability and invasion of ESCs (Fig. 6A and B).

These results described previously suggest that the abnormally low IL-24 induced by macrophages results in the increase of Ki-67, PCNA and COX-2, a decrease of
CD82 level in ESCs and the upregulation of proliferation and invasion in ESCs, and further contributes to development of endometriosis.

\section{Discussion}

Our previous research has proved that estrogen promotes proliferation and growth of DSCs by downregulating IL-24 in early pregnancy (Shao et al. 2013). In this study, we first demonstrated that the expression of IL-24 and its receptors in eutopic and ectopic ESCs derived from patients with endometriosis was decreased. The absence of IL-24 led to the increase of proliferation and invasion of ESCs in vitro. As an estrogen-dependent disease, endometriosis is defined as the presence of endometriallike tissue outside the uterine cavity, the implantation and growth of the retrograded endometrial cells into the peritoneum is a key step for endometriosis (Witz et al. 2001). Therefore, IL-24 may be also involved in the effect of estrogen on endometriosis.

After binding to its receptor dimers IL-20R1/IL-20R2 or IL-22R1/IL-20R2, MDA-7/IL-24 plays a lot of physiological and pathological functions (Dumoutier et al. 2001). Here, we found that IL-20R2 expression was also abnormally low in eutopic endometrium and ectopic lesions from patients with endometriosis. Blocking IL-24 could upregulate the proliferation of ESCs. On the contrary, rhIL-24 inhibited ESCs proliferation in a dosage-dependent manner. These data suggest that both endogenous and exogenous IL-24 play an inhibitory effect on ESCs growth. Many studies demonstrate that the suppressing effects on tumor growth are mediated mainly by IL-24-activated apoptotic pathways. IL-24 induces cell death through the activation of PKR, such as endoplasmic reticulum kinase (PERK), an unfolded protein response sensor. IL-24 can prevent the interaction between the endoplasmic reticulum (ER)residing chaperone protein BiP/GRP78 and PERK, which results in the oligomerization and autophosphorylation of PERK (Gupta et al. 2006). These events decrease the expression of pro-survival proteins (such as Mcl-1, $\mathrm{Bcl}-\mathrm{XL}, \mathrm{BCl}-2$ and $\mathrm{c}-\mathrm{Flip}$ ) and increase levels of proapoptotic markers (such as Bax and Bak) (Lebedeva et al. 2003, Su et al. 2006, Yacoub et al. 2008). Moreover, IL-24 mediates selective apoptosis in human melanoma cells by inducing the coordinated overexpression of the growth arrest and DNA damage (GADD) (Sarkar et al. 2002). Our current results show that the decrease of IL-24 in ESCs leads to the increase of Ki-67, PCNA and COX-2 of ESCs. It may also give rise to a downregulation of ESCs apoptosis.

Activation of PERK triggers the vacuolization of LC3 protein and results in an increased expression of autophagy markers (ATG5 and Beclin-1) and also induced an increased level of ROS, which leads to an increase of IL-24-mediated toxic autophagy and apoptosis (Yacoub et al. 2008). The autophagy level in 
the ectopic endometriotic tissue is decreased, and the low level of autophagy is dependent on the estrogen/ CXCL12 axis in ESCs and involved in the pathogenesis of endometriosis (Mei et al. 2015). Interestingly, IL-24 can inhibit lung tumor cells migration by disrupting the CXCL12/CXCR4 signaling pathway (Panneerselvam et al. 2015). Therefore, the absence of IL-24 in ESCs may also participate in the process of estrogen-CXCL12/ CXCR4 axis on autophagy regulation of ESCs. This area will require further research.

On this basis, we further found IL-24-promoted CD82 expression and inhibited COX-2 expression and invasiveness of ESCs in vitro. It has been reported that IL-24 also plays a role in inhibiting tumor invasion and metastasis by downregulating the expression of metastasis-related genes such as CD44, intercellular cell adhesion molecule-1 (ICAM-1), matrix metalloproteinase (MMP)-2, MMP-9, survivin, TGF- $\beta$, and the activation of Akt and NF-kB signaling pathways (Huo et al. 2013). CD82 can restrict the invasiveness of ESCs by increasing the expression of tissue inhibitor of metalloproteinase (TIMP)-1 and TIMP2 (Li et al. 2011). These actions indicate that the declining level of IL-24 may cause the enhancement of ESCs invasion by downregulating COX-2 and up-regulating CD82 and its downstream signals (such as TIMPs). However, this action of IL-24 may be independent on IDO.

The ectopic localization of endometrial tissue fragments implies survival and proliferation in a local microenvironment with a prerequisite of immune tolerance. It elicits a series of immune responses including recruitment of macrophages, blood vessels, cytokines and nerve fibers into the resultant lesions (Cao et al. 2004, Asante \& Taylor 2011, Burney \& Giudice 2012). Macrophages are functionally polarized into the classically activated macrophages phenotype (M1) and the alternatively activated macrophages phenotype (M2), which is alternatively activated in the inflammatory peritoneal fluid and in the endometriotic lesions (Bacci et al. 2009). Our and other researches have proven that macrophages are involved in ectopic adhesion, implantation, growth and vascularization of the endometriotic tissue (Bacci et al. 2009, Capobianco et al. 2011, Li et al. 2012, 2014, Mei et al. 2014), and estradiol may lead to the development of endometriosis by inducing M2 polarization of macrophages (Wang et al. 2015). Therefore, macrophages are thought to have a pivotal role in the development and maintenance of endometriosis. In this study, macrophages can downregulate IL-24 and its receptor IL-22R1 expression, and further promote proliferation and invasion of ESCs, suggesting that IL-24 may also be involved in the regulatory functions of macrophages on ESCs.

Besides regulation of ESCs invasiveness, CD82 decreases the secretion of chemokine CCL2 by ESCs, and possibly participates in controlling the recruitment of macrophages to ectopic lesions in endometriosis (Li et al. 2011). The suppression of CD82 by macrophages-IL-24 signal may stimulate the production of CCL2 by ESCs, and further induce the accumulation of macrophages in endometriotic milieu, and from a positive feedback. COX-2 and its derivative prostaglandin $\mathrm{E}_{2}\left(\mathrm{PGE}_{2}\right)$ have also been proven to play a key role in the origin and development of endometriosis through multiple mechanisms, including increased migration and invasiveness (Matsuzaki et al. 2004, Carli et al. 2009, Kao et al. 2011, Sacco et al. 2012). Previous studies have suggested that COX-2 expression is increased in the eutopic endometrium and the ovarian endometriotic tissue of patients with endometriosis (Cho et al. 2010) and its derivative PGS, particularly PGE2, can explain most of the symptoms of endometriosis (Chishima et al. 2002), especially the induction of pain. In addition, PGE2 can inhibit CD36-dependent phagocytosis of macrophages in endometriosis (Chuang et al. 2010). Therefore, macrophages increase COX-2 expression of ESCs by downregulation of IL-24 in the macrophages-ESCs co-culture unit, and may further induce the production of $\mathrm{PGE}_{2}$. In turn, $\mathrm{PGE}_{2}$ leads to the decreased scavenger activity of macrophages and further accelerates the development of endometriosis. Further experiments are needed to verify this conjecture.

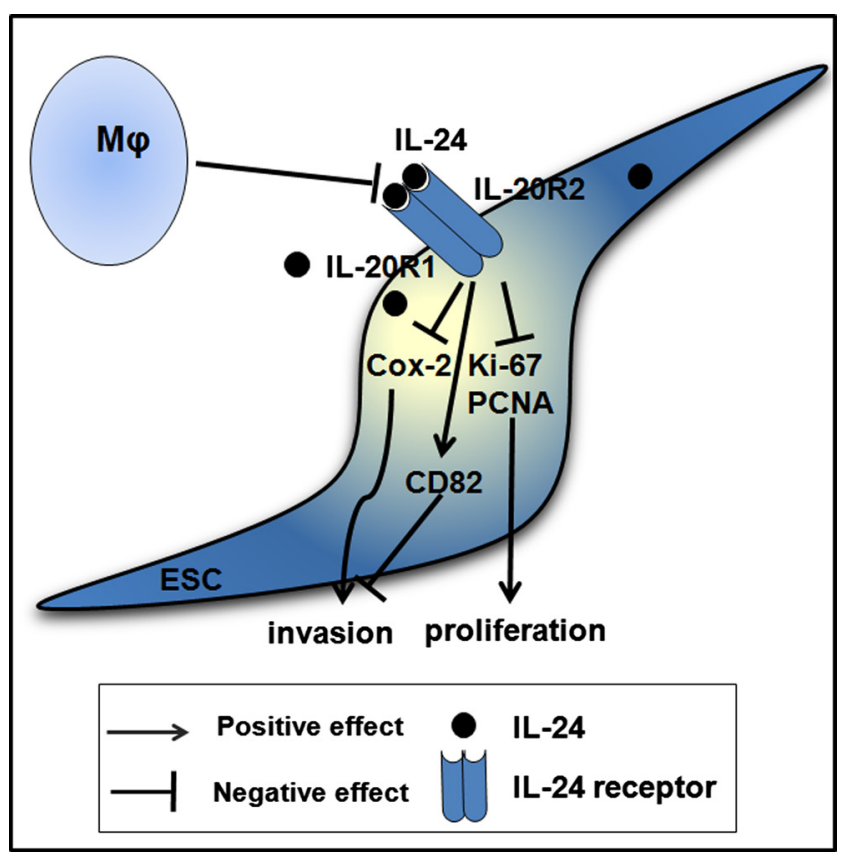

Figure 7 Schematic representation of the roles of IL-24 in the crosstalk between ESCs and macrophages in endometriosis. IL-24 inhibits the expression of Ki-67, PCNA and COX-2 and promotes the expression of CD82 in ESCs. However, macrophages can downregulate the expression of IL-24 and its receptors in ESCs, and then lead to increase in Ki-67, PCNA and COX-2, a decrease of CD82 level, and a high proliferation and invasion abilities of ESCs, and may further contribute to the survival, growth and implantation of ectopic endometrium, and finally promote the progression of endometriosis. 
Collectively, based on this study and other reports, it can be concluded that the abnormally low level of IL-24 and its receptor in ESCs possibly induced by macrophages and or local high level of estrogen, on the one hand, leads to the increase of Ki-67, PCNA and COX-2 and a decrease of the CD82 level, and further stimulates the proliferation and invasion of ESCs (Fig. 7); on the other hand, this probably results in the recruitment of macrophages by CD82/CCL2 signal, and the impaired phagocytosis of macrophage by $\mathrm{COX}-2 / \mathrm{PGE}_{2}$ axis, which finally contributes to the survival, growth and implantation of ectopic endometrium and promotes the progress of endometriosis. IL-24 may be a biomarker for the diagnosis and treatment for endometriosis. These potential values are worth further investigation.

\section{Declaration of interest}

The authors declare that there is no conflict of interest that could be perceived as prejudicing the impartiality of the research reported.

\section{Funding}

This study was supported by the Major Research Program of the National Natural Science Foundation of China (NSFC) 91542108, the NSFC 81471513, the Shanghai RisingStar Program (16QA1400800), the Training Program for Young Talents of Shanghai Health System XYQ2013104, the Development Fund of Shanghai Talents (201557) and the Program for Zhuoxue of Fudan University (all to M-Q Li); the NSFC 81270677 (to X-Y Zhu); the NSFC 81571509 (to J Shao); the Program for Shanghai leaders, the Program of Shanghai Outstanding Academic Leader (15XD1500900) and the Training Program for excellent academic leaders of Shanghai Health System (XBR2013093) (to L-P Jin).

\section{References}

Asante A \& Taylor RN 2011 Endometriosis: the role of neuroangiogenesis. Annual Review of Physiology 73 163-182. (doi:10.1146/annurevphysiol-012110-142158)

Bacci M, Capobianco A, Monno A, Cottone L, Di Puppo F, Camisa B, Mariani M, Brignole C, Ponzoni M, Ferrari S et al. 2009 Macrophages are alternatively activated in patients with endometriosis and required for growth and vascularization of lesions in a mouse model of disease. American Journal of Pathology 175 547-556. (doi:10.2353/ ajpath.2009.081011)

Burney RO \& Giudice LC 2012 Pathogenesis and pathophysiology of endometriosis. Fertility and Sterility 98 511-519. (doi:10.1016/j. fertnstert.2012.06.029)

Cao X, Yang D, Song M, Murphy A \& Parthasarathy S 2004 The presence of endometrial cells in the peritoneal cavity enhances monocyte recruitment and induces inflammatory cytokines in mice: implications for endometriosis. Fertility and Sterility 82 999-1007. (doi:10.1016/j. fertnstert.2004.04.040)

Capobianco A, Monno A, Cottone L, Venneri MA, Biziato D, Di Puppo F, Ferrari S, De Palma M, Manfredi AA \& Rovere-Querini P 2011 Proangiogenic Tie2(+) macrophages infiltrate human and murine endometriotic lesions and dictate their growth in a mouse model of the disease. American Journal of Pathology 179 2651-2659. (doi:10.1016/j. ajpath.2011.07.029)
Carli C, Metz CN, Al-Abed Y, Naccache PH \& Akoum A. 2009 Up-regulation of cyclooxygenase-2 expression and prostaglandin E2 production in human endometriotic cells by macrophage migration inhibitory factor: involvement of novel kinase signaling pathways. Endocrinology 150 3128-3137. (doi:10.1210/en.2008-1088)

Chishima F, Hayakawa S, Sugita K, Kinukawa N, Aleemuzzaman S, Nemoto N, Yamamoto T \& Honda M 2002 Increased expression of cyclooxygenase-2 in local lesions of endometriosis patients. American Journal of Reproductive Immunology 48 50-56. (doi:10.1034/j.16000897.2002.01101.x)

Cho S, Park SH, Choi YS, Seo SK, Kim HY, Park KH, Cho DJ \& Lee BS 2010 Expression of cyclooxygenase-2 in eutopic endometrium and ovarian endometriotic tissue in women with severe endometriosis. Gynecologic and Obstetric Investigation 69 93-100. (doi:10.1159/000261017)

Chuang PC, Lin YJ, Wu MH, Wing LY, Shoji Y \& Tsai SJ 2010 Inhibition of CD36-dependent phagocytosis by prostaglandin E2 contributes to the development of endometriosis. American Journal of Pathology 176 850-860. (doi:10.2353/ajpath.2010.090551)

Dent P, Yacoub A, Hamed HA, Park MA, Dash R, Bhutia SK, Sarkar D, Wang XY, Gupta P, Emdad L et al. 2010 The development of MDA-7/IL24 as a cancer therapeutic. Pharmacology \& Therapeutics 128 375-384. (doi:10.1016/j.pharmthera.2010.08.001)

Dumoutier L, Leemans C, Lejeune D, Kotenko SV \& Renauld JC 2001 Cutting edge: STAT activation by IL-19, IL-20 and mda-7 through IL-20 receptor complexes of two types. Journal of Immunology $\mathbf{1 6 7}$ 3545-3549. (doi:10.4049/jimmunol.167.7.3545)

Dunselman GA, Hendrix MG, Bouckaert PX \& Evers JL 1988 Functional aspects of peritoneal macrophages in endometriosis of women. Journal of Reproduction \& Infertility 82 707-710. (doi:10.1530/jrf.0.0820707)

Fisher PB, Gopalkrishnan RV, Chada S, Ramesh R, Grimm EA, Rosenfeld MR, Curiel DT \& Dent P 2003 mda-7/IL-24, a novel cancer selective apoptosis inducing cytokine gene: from the laboratory into the clinic. Cancer Biology \& Therapy 2 S22-S36. (doi:10.4161/cbt.458)

Giudice LC 2010 Clinical practice. Endometriosis. New England Journal of Medicine 362 2389-2398. (doi:10.1056/NEJMcp1000274)

Giudice LC \& Kao LC 2004 Endometriosis. Lancet 364 1789-1799. (doi:10.1016/S0140-6736(04)17403-5)

Gupta P, Walter MR, Su ZZ, Lebedeva IV, Emdad L, Randolph A, Valerie K, Sarkar D \& Fisher PB 2006 BiP/GRP78 is an intracellular target for MDA-7/IL-24 induction of cancer-specific apoptosis. Cancer Research 66 8182-8191. (doi:10.1158/0008-5472.CAN-06-0577)

Halme J, Becker S \& Wing R 1984 Accentuated cyclic activation of peritoneal macrophages in patients with endometriosis. American Journal of Obstetrics \& Gynecology 148 85-90. (doi:10.1016/S00029378(84)80037-X)

Halme J, Becker S \& Haskill S 1987 Altered maturation and function of peritoneal macrophages: possible role in the pathogenesis of endometriosis. American Journal of Obstetrics \& Gynecology 156 783-789. (doi:10.1016/0002-9378(87)90333-4)

Haney AF, Muscato JJ \& Weinberg JB 1981 Peritoneal fluid cell populations in infertility patients. Fertility and Sterility 35 696-698. (doi:10.1016/ S0015-0282(16)45567-6)

Huo W, Li ZM, Zhu XM, Bao YM \& An LJ 2013 MDA-7/IL-24 suppresses tumor adhesion and invasive potential in hepatocellular carcinoma cell lines. Oncology Reports 30 986-992. (doi:10.3892/or.2013.2507)

Kao AP, Wang KH, Long CY, Chai CY, Tsai CF, Hsieh TH, Hsu CY, Chang CC, Lee JN \& Tsai EM 2011 Interleukin-1 $\beta$ induces cyclooxygenase-2 expression and promotes the invasive ability of human mesenchymal stem cells derived from ovarian endometrioma. Fertility and Sterility 96 678-684.e1. (doi:10.1016/j.fertnstert.2011.06.041)

Lebedeva IV, Sarkar D, Su ZZ, Kitada S, Dent P, Stein CA, Reed JC \& Fisher PB 2003 Bcl-2 and Bcl-x(L) differentially protect human prostate cancer cells from induction of apoptosis by melanoma differentiation associated gene-7, mda-7/IL-24. Oncogene 22 8758-8773. (doi:10.1038/sj.onc.1206891)

Li MQ, Hou XF, Lv SJ, Meng YH, Wang XQ, Tang CL \& Li DJ 2011 CD82 gene suppression in endometrial stromal cells leads to increase of the cell invasiveness in the endometriotic milieu. Journal of Molecular Endocrinology 47 195-208. (doi:10.1530/JME-10-0165)

Li MQ, Li HP, Meng YH, Wang XQ, Zhu XY, Mei J \& Li DJ 2012 Chemokine CCL2 enhances survival and invasiveness of endometrial stromal cells in an autocrine manner by activating Akt and MAPK/Erk1/2 signal pathway. Fertility and Sterility 97 919-929. (doi:10.1016/j.fertnstert.2011.12.049) 
Li MQ, Wang Y, Chang KK, Meng YH, Liu LB, Mei J, Wang Y, Wang XQ, Jin LP \& Li DJ $2014 \mathrm{CD}^{+}{ }^{+} \mathrm{Foxp}^{+}$regulatory $\mathrm{T}$ cell differentiation mediated by endometrial stromal cell-derived TECK promotes the growth and invasion of endometriotic lesions. Cell Death \& Disease 5 e1436. (doi:10.1038/cddis.2014.414)

Matsuzaki S, Canis M, Darcha C, Dallel R, Okamura K \& Mage G 2004 Cyclooxygenase-2 selective inhibitor prevents implantation of eutopic endometrium to ectopic sites in rats. Fertility and Sterility 82 1609-1615. (doi:10.1016/j.fertnstert.2004.07.946)

Mei J, Li MQ, Ding D, Li DJ, Jin LP, Hu WG \& Zhu XY 2013 Indoleamine 2,3-dioxygenase-1 (IDO1) enhances survival and invasiveness of endometrial stromal cells via the activation of JNK signaling pathway. International Journal of Clinical and Experimental Pathology 6 431-444.

Mei J, Xie XX, Li MQ, Wei CY, Jin LP, Li DJ \& Zhu XY 2014 Indoleamine 2,3-dioxygenase-1 (IDO1) in human endometrial stromal cells induces macrophage tolerance through interleukin-33 in the progression of endometriosis. International Journal of Clinical and Experimental Pathology 7 2743-2757.

Mei J, Zhu XY, Jin LP, Duan ZL, Li DJ \& Li MQ 2015 Estrogen promotes the survival of human secretory phase endometrial stromal cells via CXCL12/CXCR4 up-regulation-mediated autophagy inhibition. Human Reproduction 30 1677-1689. (doi:10.1093/humrep/dev100)

Meuleman C, Vandenabeele B, Fieuws S, Spiessens C, Timmerman D \& D'Hooghe T 2009 High prevalence of endometriosis in infertile women with normal ovulation and normospermic partners. Fertility and Sterility 92 68-74. (doi:10.1016/j.fertnstert.2008.04.056)

Panneerselvam J, Jin J, Shanker M, Lauderdale J, Bates J, Wang Q, Zhao YD, Archibald SJ, Hubin TJ \& Ramesh R 2015 IL-24 inhibits lung cancer cell migration and invasion by disrupting the SDF-1/CXCR4 signaling axis. PLOS ONE 10 e0122439. (doi:10.1371/journal.pone.0122439)

Sacco K, Portelli M, Pollacco J, Schembri-Wismayer P \& Calleja-Agius J 2012 The role of prostaglandin E2 in endometriosis. Gynecological Endocrinology 28 134-138. (doi:10.3109/09513590.2011.588753)

Sampson JA 1927 Metastatic or embolic endometriosis, due to the menstrual dissemination of endometrial tissue into the venous circulation. American Journal of Pathology 3 93-110.43.

Sarkar D, Su ZZ, Lebedeva IV, Sauane M, Gopalkrishnan RV, Valerie K, Dent P \& Fisher PB 2002 mda-7 (IL-24) Mediates selective apoptosis in human melanoma cells by inducing the coordinated overexpression of the GADD family of genes by means of p38 MAPK. PNAS 99 10054-10059. (doi:10.1073/pnas.152327199)

Sauane M, Gopalkrishnan RV, Sarkar D, Su ZZ, Lebedeva IV, Dent P, Pestka S \& Fisher PB 2003 MDA-7/IL-24: novel cancer growth suppressing and apoptosis inducing cytokine. Cytokine \& Growth Factor Reviews 14 35-51. (doi:10.1016/S1359-6101(02)00074-6)
Sauane M, Su ZZ, Gupta P, Lebedeva IV, Dent P, Sarkar D \& Fisher PB 2008 Autocrine regulation of mda-7/IL-24 mediates cancer-specific apoptosis. PNAS 105 9763-9768. (doi:10.1073/pnas.0804089105)

Shao J, Li MQ, Meng YH, Chang KK, Wang Y, Zhang L \& Li DJ 2013 Estrogen promotes the growth of decidual stromal cells in human early pregnancy. Molecular Human Reproduction 19 655-664. (doi:10.1093/ molehr/gat034)

Su ZZ, Lebedeva IV, Sarkar D, Gopalkrishnan RV, Sauane M, Sigmon C, Yacoub A, Valerie K, Dent P \& Fisher PB 2003 Melanoma differentiation associated gene-7, mda-7/IL-24, selectively induces growth suppression, apoptosis and radiosensitization in malignant gliomas in a p53-independent manner. Oncogene 22 1164-1180. (doi:10.1038/ sj.onc.1206062)

Su ZZ, Lebedeva IV, Sarkar D, Emdad L, Gupta P, Kitada S, Dent P, Reed JC \& Fisher PB 2006 lonizing radiation enhances therapeutic activity of mda-7/IL-24: overcoming radiation- and mda-7/IL-24-resistance in prostate cancer cells overexpressing the antiapoptotic proteins bcl-xL or bcl-2. Oncogene 25 2339-2348. (doi:10.1111/j.16000897.2009.00750.x)

Ueda M, Yamashita Y, Takehara M, Terai Y, Kumagai K, Ueki K, Kanda K, Yamaguchi H, Akise D, Hung YC et al. 2002 Survivin gene expression in endometriosis. Journal of Clinical Endocrinology \& Metabolism 87 3452-3459. (doi:10.1210/jc.87.7.3452)

Wang Y, Chen H, Wang N, Guo H, Fu Y, Xue S, Ai A, Lyu Q \& Kuang Y 2015 Combined $17 \beta$-estradiol with TCDD promotes M2 polarization of macrophages in the endometriotic milieu with aid of the interaction between endometrial stromal cells and macrophages. PLOS ONE 10 e0125559. (doi:10.1371/journal.pone.0125559)

Witz CA, Thomas MR, Montoya-Rodriguez IA, Nair AS, Centonze VE \& Schenken RS 2001 Short-term culture of peritoneum explants confirms attachment of endometrium to intact peritoneal mesothelium. Fertility and Sterility 75 385-390. (doi:10.1016/S0015-0282(00)01699-X)

Yacoub A, Park MA, Gupta P, Rahmani M, Zhang G, Hamed H, Hanna D, Sarkar D, Lebedeva IV, Emdad L et al. 2008 Caspase-, cathepsin-, and PERK-dependent regulation of MDA-7/IL-24-induced cell killing in primary human glioma cells. Molecular Cancer Therapeutics 7 297-313. (doi:10.1158/1535-7163)

Received 18 May 2016

First decision 14 July 2016

Revised manuscript received 11 August 2016

Accepted 13 September 2016 